\title{
Magnetorheological photocatalytic systems
}

\author{
D. G. Shchukin, ${ }^{1}$ D. V. Sviridov, ${ }^{1}$ and A. I. Kulak ${ }^{2}$ \\ ${ }^{1}$ Institute for Physico-Chemical Problems, Belarusian State University, \\ Leningradskaya 14, 220050 Minsk, Belarus \\ ${ }^{2}$ Institute of General and Inorganic Chemistry, Belarusian Academy \\ of Sciences, Surganova 9, 220072 Minsk, Belarus
}

\begin{abstract}
Preparation and properties of a novel photocatalytic system containing magnetic cores coated with subsequently applied silica and titania shells are discussed. The underlying idea is to impart magnetic properties to the semiconductor particles that permits to control the rheological properties of the photocatalyst dispersion and makes possible its separation from treated solution without invoking procedures of filtration or centrifugation. Preparation route yielding photoactive titania coating and silica interlayer, which prevents the undesirable doping of catalyst and parasitic charge exchange between titania shell and magnetic core, is described in detail.
\end{abstract}

\section{INTRODUCTION}

In recent years, a great deal of attention has been drawn toward a photocatalytic technologies including a semiconductor-sensitised photocatalytic detoxification of aquatic environments [1-3]. The interest in the photocatalytic detoxification is mainly due to the fact that the degradation of organic pollutants in the illuminated suspensions and colloids of semiconductor oxides (such as $\mathrm{TiO}_{2}$ ) generally leads to the complete mineralization of organics being not accompanied, in contrast to the conventional detoxification techniques, with the formation of xenobiotic byproducts. The chief disadvantage of slurry-based photocatalytic reactor systems is the need to ultimate remove semiconductor particles from treated solution by filtration or centrifugation that complicates the photocatalytic degradation technologies to such extent that their economic viability in many cases becomes questionable. To eliminate the need for additional steps of post-process filtration and to realize the continuous photocatalytic processing of aqueous effluents, a variety of photocatalytic systems employing semiconductor photocatalysts immobilized onto conductive $[4,5]$ or non-conductive $[3,6]$ supports (including an internal lightguide [7]) were proposed. However, the fixed-bed photocatalytic reactors also suffer from many drawbacks; most notable are the low surface area-to volume ratios, sensitivity to fouling and inefficiencies induced by absorption of actinic light by the reaction media.

In order to resolve these problems, a magnetic microheterogeneous catalysts of core-shell type were devised as a photocatalyst system for decomposing aquatic pollutants as well as for other photochemical applications. The dispersed magnetic photocatalysts, which combine properties of ferromagnetic fluid and nonmagnetic semiconductor dispersion, can be readily removed from treated solution and are expected to utilize light with higher efficiency as compared to particulate semiconductor films in the fixed-bed photocatalytic re- actor. This paper describes the characterization of the $\mathrm{TiO}_{2}$ photocatalyst with a magnetic core and results of the evaluation of its photocatalytic efficiency.

\section{MATERIALS AND METHODS}

As the magnetic cores, dispersions of ferromagnetic perovskite $\mathrm{Li}_{0.05} \mathrm{La}_{0.5} \mathrm{Sr}_{0.4} \mathrm{Mn}_{0.95} \mathrm{O}_{3}$ (prepared by sintering of corresponding oxides) and spinel ferrite $\mathrm{CoFe}_{2} \mathrm{O}_{4}$ (prepared via co-precipitation from cobaltous and ferric chlorides in basic medium followed by annealing of the obtained powder at $450^{\circ} \mathrm{C}$ [8]) were used. In both cases, the size of magnetic particles was of $\sim 1 \mu \mathrm{m}$. Titania coating was deposited onto these magnetic microbeads by hydrolysis of titanium alkoxide precursor; for this purpose, the dispersion to be modified (8 g/l) was mechanically (i.e., "nonmagnetically") stirred for 1.5 hours in the ethanol solution containing water ( $\sim 4$ volume percent) and titanium- $n$-butoxide $(9.5 \mathrm{mM})$. Titania-modified particles were then precipitated in the magnetic field, washed with ethanol and annealed at $450{ }^{\circ} \mathrm{C}$. The $\mathrm{TiO}_{2}$ loading obtained by means of emission analysis corresponds to a titania film $5-7 \mathrm{~nm}$ in thickness. The deposition of silica coating onto dispersed manganite (activated by keeping in $0.1 \mathrm{M} \mathrm{NaOH}$ overnight) and ferrite (used as prepared) resembles the Stöber process [9] modified according to ref. [10] and involves the following two steps:

(i) adsorption of thin adhesive $\mathrm{SiO}_{2}$ layer from aqueous solution of silicic acid and

(ii) growth of silica coating from non-aqueous solution. First a 0.58 wt $\left(\mathrm{SiO}_{2}\right) \%$ sodium silicate solution of $\mathrm{pH}=11.9$ was passed through a freshly regenerated acid exchange column.

To prevent silica nucleation, the $\mathrm{pH}$ of obtained eluate was then adjusted to $\mathrm{pH}=9.6$ by adding a small amount of sodium silicate solution. A $25 \mathrm{ml}$ of thus prepared solution of active silica was added to the magnetic dispersion ( $1.25 \mathrm{~g}$ in $125 \mathrm{ml}$ of water). After lowering the $\mathrm{pH}$ of solution by slow titration with $0.5 \mathrm{M}$ $\mathrm{HCl}$ until $\mathrm{pH}=10$, mechanically stirred dispersion was left for 2 hours. Further silica growth on thus modi- 
fied magnetic cores was performed via hydrolysis of tetraethoxisilane (TES). For this purpose, a mixture containing $145 \mathrm{ml}$ ethanol $(96 \%)+0.44 \mathrm{ml} \mathrm{TES}+5.1 \mathrm{ml}$ ammonia $(25 \%)+1.25$ g magnetic dispersion with adhesive silica coating was stirred for one day (manganite core) or for 12 hours (ferrite core). Thus obtained silicamodified manganite particles, after precipitation in the magnetic field and washing with ethanol, were at once used for preparation of magnetic $\mathrm{TiO}_{2}$-catalyst via the procedure given above, while the silica-modified ferrite particles were annealed at $450{ }^{\circ} \mathrm{C}$ and the procedure of growing of silica shell via hydrolysis of TES was continued for next 12 hours.

Photocatalytic investigations were carried out by using a medium pressure $120 \mathrm{~W}$ mercury lamp. The light flux incident upon the quartz window of photocatalytic cell was completely absorbed in the magnetically stirred suspension of photocatalyst $(6 \mathrm{~g} / \mathrm{l})$. To probe the efficiency of hole-transfer processes of the magnetic photocatalyst, the reaction of photodecomposition of oxalate was employed. In these experiments, aerated aqueous solution containing $5 \mathrm{mM}$ sodium oxalate $+5 \mathrm{mM}$ oxalic acid was used. The rate of disappearance of oxalate was determined by periodically running a cycling voltammogram at a carbon paste electrode and measuring the anodic peak current $(\mathrm{Ep}=+1.25 \mathrm{~V} v$ s. SCE) which is known to be directly proportional to the concentration of oxalate [11].

\section{RESULTS AND DISCUSSION}

Figure 1 shows reduction of oxalate concentration with the illumination time for magnetically stirred aqueous suspensions of several titania-coated magnetic photocatalysts. It is seen from Figure 1 that the photoreactivity of catalysts obtained by growing titania shell directly on the magnetic core is considerably lower as compared to suspension of microcrystalline titanium dioxide Sachtleben Hombikat UV 100 (pure anatase). The poor efficiently of photocatalysts $\mathrm{CoFe}_{2} \mathrm{O}_{4} / \mathrm{TiO}_{2}$ and $\mathrm{Li}_{0.05} \mathrm{La}_{0.5} \mathrm{Sr}_{0.4} \mathrm{Mn}_{0.95} \mathrm{O}_{3} / \mathrm{TiO}_{2}$ can be attributed to trapping of photogenerated carriers at the trapping sites produced in the titania lattice due to metal ion doping during annealing of photocatalyst or to the direct injection of charge carriers from photocatalytic shell into the core that shunts the charge carriers away from solid-solution interface and promotes recombination with a net reduction in photoreactivity. The separation of $\mathrm{TiO}_{2}$ catalyst from magnetic core by applying a silica interlayer considerably improves the photoreactivity: under conditions of full absorption of incident light in the stirred suspension of photocatalyst, the rate of disappearance of oxalate at microheterogeneous catalyst $\mathrm{Li}_{0.05} \mathrm{La}_{0.5} \mathrm{Sr}_{0.4} \mathrm{Mn}_{0.95} \mathrm{O}_{3} / \mathrm{SiO}_{2} / \mathrm{TiO}_{2}$ appears to be compatible to that provided by dispersed $\mathrm{TiO}_{2}$ Figure 1 .

The fact that X-ray photoelectron spectrum for magnetic photocatalyst $\mathrm{Li}_{0.05} \mathrm{La}_{0.5} \mathrm{Sr}_{0.4} \mathrm{Mn}_{0.95} \mathrm{O}_{3} / \mathrm{SiO}_{2} / \mathrm{TiO}_{2}$ shows only the photoelectron and Auger peaks char-
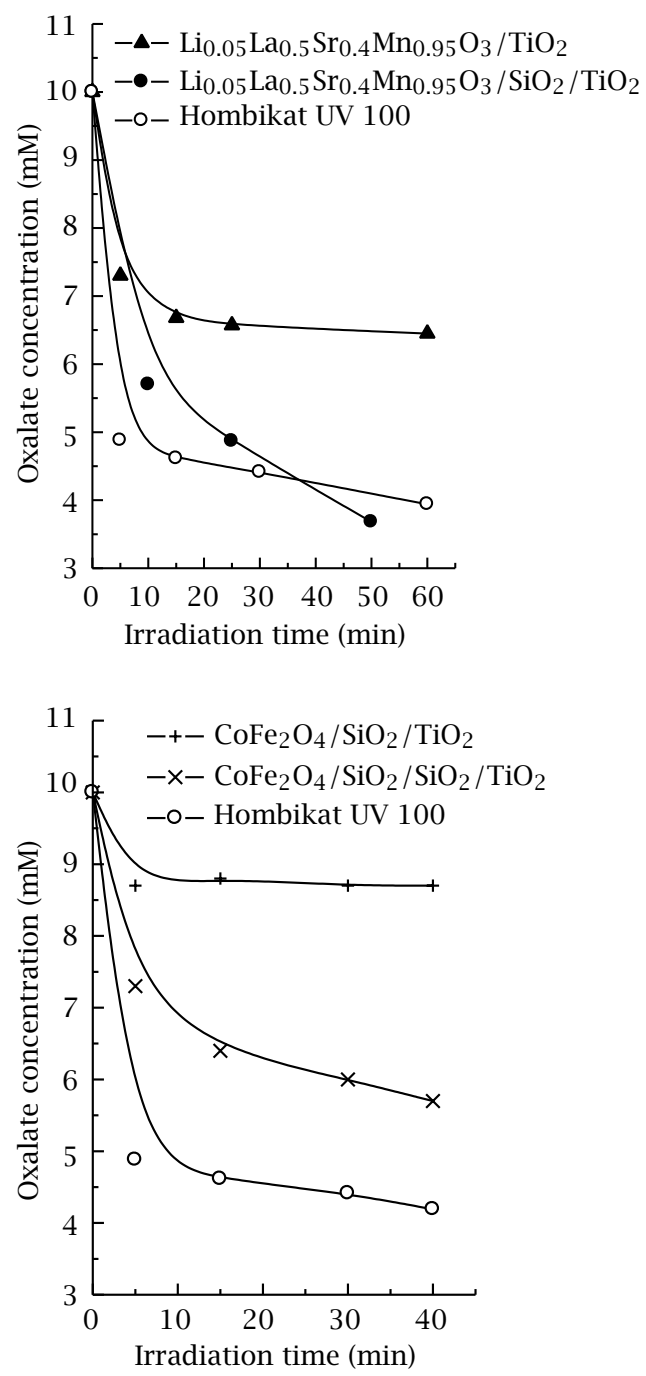

Figure 1. Time dependencies of oxalate photodegradation in the stirred suspensions of magnetic and nonmagnetic $\mathrm{TiO}_{2}$ photocatalysts.

acteristic of $\mathrm{TiO}_{2}$ and $\mathrm{SiO}_{2}$ Figure 2 is an indicative of complete encapsulation of magnetic core with silica and titania shells. The relatively high intensity of $\mathrm{Si} 2 p$ peak can be attributed to some coalescence of titania layer upon annealing associated with density changes accompanying $\mathrm{TiO}_{2}$ crystallization; as the result, the $\mathrm{TiO}_{2}$ shell may not completely cover the silica beads with an embedded magnetic core. Similar coagulation effects were observed previously for crystallization of titania coatings deposited on the Stöber silica spheres [12].

In case of $\mathrm{CoFe}_{2} \mathrm{O}_{4}$ magnetic core, the application of silica interlayer leads to only a modest increase in the photoreactivity. Taking into consideration that even a slight doping of $\mathrm{TiO}_{2}$ with $\mathrm{Co}^{3+}$ may result in a drastic decrease in the efficiency of valence band hole oxidation reactions [13], it may be suggested that the annealing of structure $\mathrm{CoFe}_{2} \mathrm{O}_{4} / \mathrm{SiO}_{2} / \mathrm{TiO}_{2}$ is accompanied with diffusion of cobalt ions through the $\mathrm{SiO}_{2}$ interlayer or with a cobalt silicate formation. This 


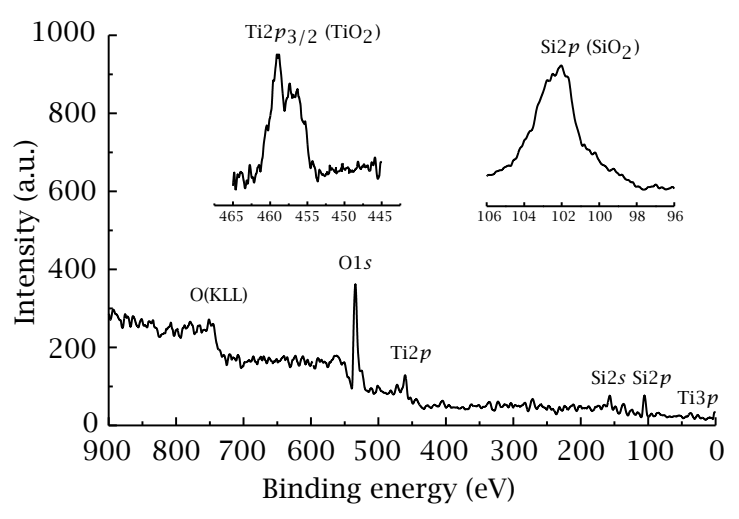

Figure 2. X-ray photoelectron spectrum for magnetic photocatalyst $\mathrm{Li}_{0.05} \mathrm{La}_{0.5} \mathrm{Sr}_{0.4} \mathrm{Mn}_{0.95} \mathrm{O}_{3} / \mathrm{SiO}_{2} / \mathrm{TiO}_{2}$ A lower binding energy shoulder to the Ti2p photoelectron peak is due to the non-stoichiometric titanium oxide.

suggestion is supported by a fact that the photocatalyst reactivity can be vastly enhanced Figure 1 if the deposition of silica shell is interrupted and the $\mathrm{SiO}_{2}$-modified cobalt ferrite particles are heated at $450{ }^{\circ} \mathrm{C}$; this twostep procedure of deposition of silica coating permits to suppress further penetration of cobalt ions through the $\mathrm{SiO}_{2}$ interlayer during the course of final annealing of photocatalyst.

\section{CONCLUSIONS}

The principles of preparation of magneticallycontrollable $\mathrm{TiO}_{2}$ photocatalyst of core-shell type with embedded magnetic particles were developed. The magnetorheological properties of the photocatalytic systems of this sort make possible the straightforward separation of photocatalyst from treated solution and thus allow for continuous use of photocatalyst for processing aqueous effluents while eliminating the need for post-process filtration. Furthermore, the magnetic properties of these photocatalysts permit to exert an efficient control over the reaction zone of photoreactor.

\section{ACKNOWLEDGEMENTS}

This work was supported by UNESCO under Grant BYE 315. D.G.S. also acknowledges support from the Basic Research Foundation of Belarus.

\section{REFERENCES}

[1] M. Hoffmann, S. Martin, W. Choi, and D. Bahnemann, Chem. Rev. 95 (1995), 69.

[2] D. F. Ollis and H. Al-Ekabi, Photocatalytic Purification and Treatment of Water and Air, Elsevier, Amsterdam, 1993.

[3] I. Rosenberg, J. R. Brock, and A. Heller, J. Phys. Chem. 96 (1992), 3423.

[4] H. Hidaka, Y. Asai, J. Zhao, K. Nohara, E. Pelizzetti, and N. Serpone, J. Phys. Chem. 99 (1995), 8244.

[5] K. Vinodgopal, S. Hotchandani, and P. V. Kamat, J. Phys. Chem. 97 (1993), 9040.

[6] R. W. Matthews, J. Phys. Chem. 91 (1987), 3328.

[7] H. Tada and H. Honda, J. Electrochem. Soc. 142 (1995), 3438.

[8] G. I. Traikovskaya, R. M. Potapenko, and V. V. Sviridov, Izv. AN BSSR (1965), 52 (Russian).

[9] W. Stöber, A. Fink, and E. Bohn, J. Colloid Interface Sci. 26 (1968), 62.

[10] A. P. Philipse, M. P. B. van Bruggen, and C. Pathmamanoharan, Langmuir 10 (1994), 92.

[11] K. V. Thrivikraman, R. W. Keller, S. K. Wolfson, S. J. Yao, and J. C. Morgenlander, Bioelectrochem. Bioenerg. 9 (1982), 357.

[12] A. Hanprasopwattana, S. Srinivasan, A. G. Sault, and A. K. Datye, Langmuir 12 (1996), 3173.

[13] W. Choi, A. Termin, and M. R. Hoffmann, J. Phys. Chem. 98 (1994), 13669. 


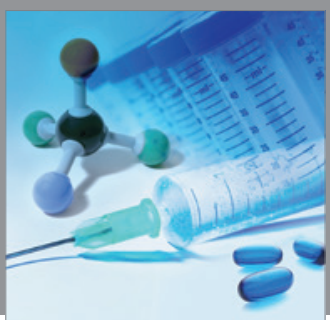

International Journal of

Medicinal Chemistry

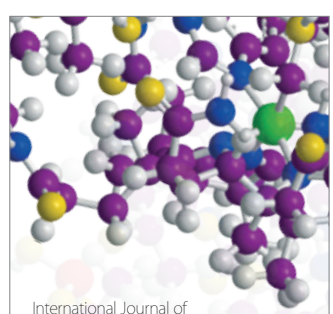

Carbohydrate Chemistry

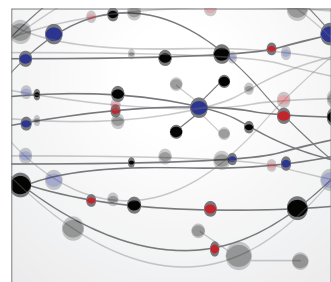

The Scientific World Journal
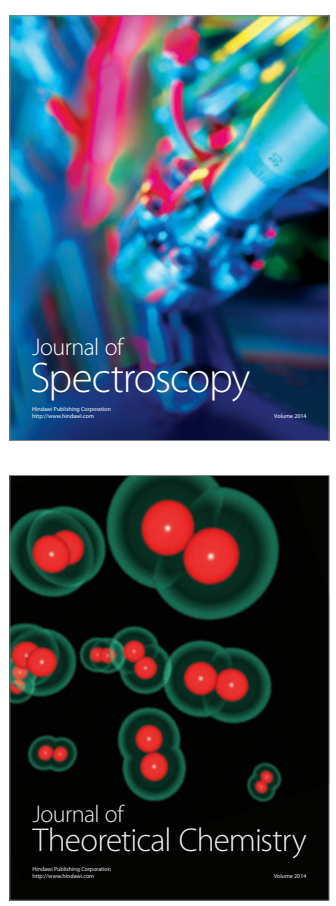
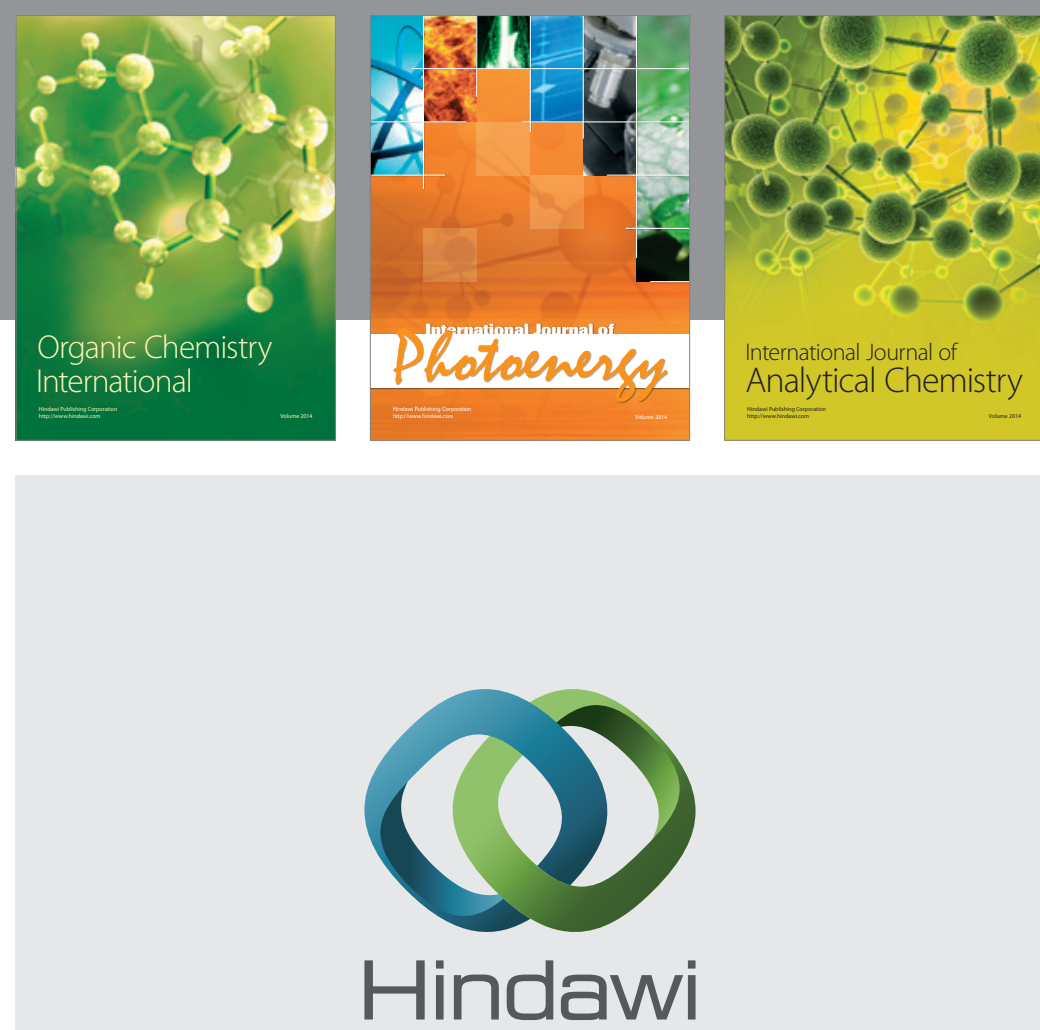

Submit your manuscripts at

http://www.hindawi.com
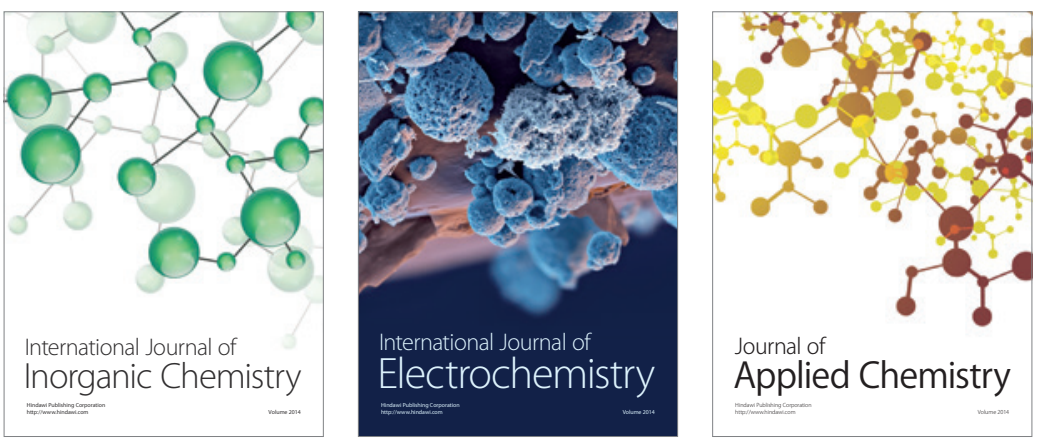

Journal of

Applied Chemistry
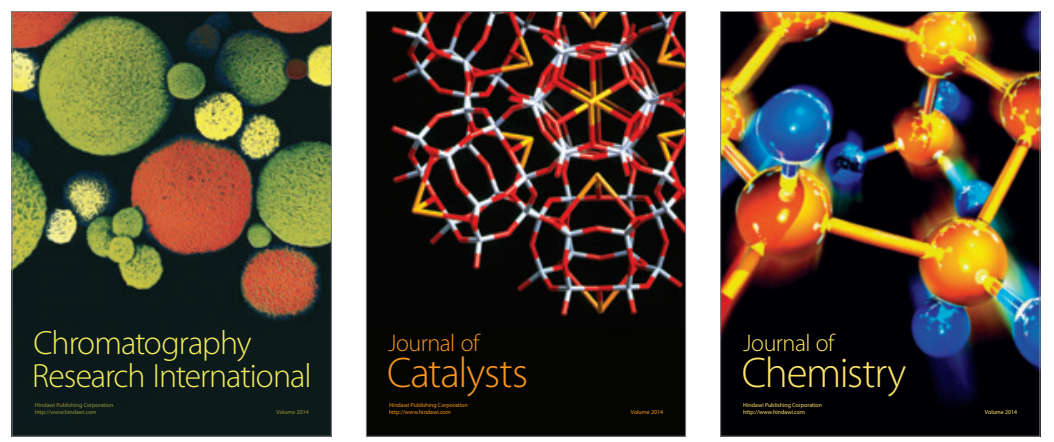
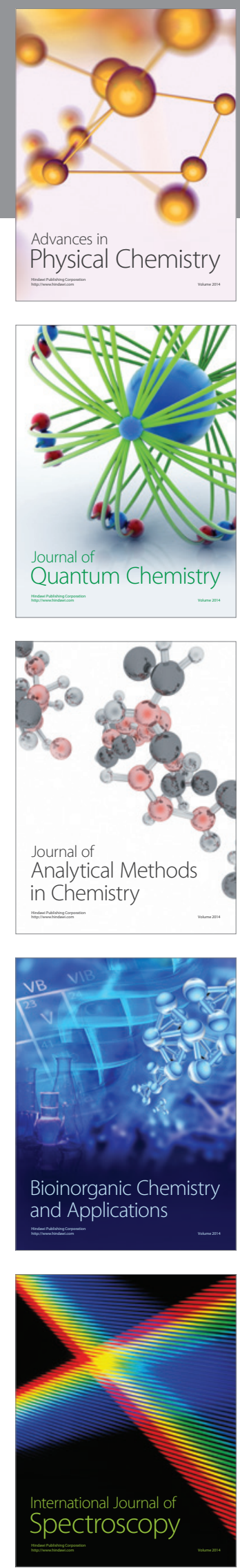